\title{
Attentionale Horizonte zwischen Phänomenologie und Psychologie
}

Maren Wehrle und Thiemo Breyer

\section{Einleitung}

Die psychologische wie auch die philosophische Untersuchung der Aufmerksamkeit nimmt ihren Ausgang von der Problematisierung des Umstands, dass wir uns nicht allen wahrnehmbaren Dingen gleichermaßen bzw. mit der gleichen Bewusstseinsqualität zuwenden können. Insofern muss eine nach bestimmten Kriterien verlaufende Auswahl stattfinden. Die psychologische Aufmerksamkeitsforschung fasst diese Tendenz mit dem Konzept der Selektion, das eng mit der Annahme zusammenhängt, dass die menschliche Informationsverarbeitung beschränkt ist. Der angenommene Selektionsmechanismus filtert diejenigen Reize, Orte oder Gegenstände heraus, die nicht eingehender verarbeitet werden sollen, denen somit also keine Aufmerksamkeit zuteil wird. Einer ähnlichen Beschränkungslogik folgt die Annahme von Aufmerksamkeit als einer begrenzten Kapazität oder Ressource.

Stellt aktiv auswählende Aufmerksamkeit ein kontinuierliches Motiv der psychologischen Aufmerksamkeitsforschung dar, gilt dies gleichermaßen für ihre passive Seite, die in vielerlei Gestalten auftritt. Als passiv lassen sich etwa sowohl die Voraussetzungen, Motivationen und äußeren Ursachen eines aktuellen Aufmerksamkeitsaktes verstehen, als auch dessen unbemerkter gegenständlicher Hintergrund. In den Anfängen der Psychologie als wissenschaftliche Disziplin findet sich die Differenzierung in eine willentliche (aktive) und eine unwillentliche (passive) Form der Aufmerksamkeit etwa bei William James und Edward Titchener. Als erste Stufe der Aufmerksamkeit wird hier eine rezeptive Bereitschaft für neue Wahrnehmungsstimuli gefasst: Erst wenn neue Reize untereinander konkurrieren, kommt es in einem zweiten Schritt zur aktiven, willentlich auswählenden Aufmerksamkeit. Das passive Bemerken eines Donnergrollens (primäre Aufmerksamkeit) 
ist insofern von einer expliziten Auseinandersetzung mit einem mathematischen Satz (sekundäre Aufmerksamkeit) zu unterscheiden. ${ }^{1}$

Rückblickend erscheinen diese frühen Konzeptionen als Vorgänger der später in der Kognitions- und Neuropsychologie geläufigen Unterscheidung zwischen einer bottom-up (reizbasiert) und top-down (intentional) regulierten Steuerung der Aufmerksamkeit. Im Gegensatz zu einer strikten Trennung zwischen beiden Arten der Aufmerksamkeitslenkung macht Titchener bereits die Interdependenz der beiden Ebenen deutlich: Der Vorgang der sekundären Selektion kann nicht isoliert verstanden werden, sondern ist auf einen bereits bestehenden passiv-sensorischen Untergrund angewiesen. Es handelt sich insofern zwar um unterschiedliche Komplexitätsstufen und Phasen des Aufmerksamkeitsgeschehens, nicht aber um isolierte, voneinander unabhängige Systeme. Dennoch gibt es in der psychologischen ebenso wie in der philosophischen Forschung zahlreiche Versuche, Aufmerksamkeit entweder als subjektive willentliche Leistung einerseits oder als von außen bedingte automatische Ausrichtung andererseits zu definieren. Eine eindeutige Zuschreibung lässt sich jedoch nur in spezifischen Fällen (etwa in einem klar eingegrenzten experimentellen Kontext) und auch dann nur in eingeschränktem Maße vornehmen.

Anstatt sich argumentativ oder konzeptuell auf die eine oder andere Seite zu schlagen, wie dies vor allem in der heutigen Kognitionspsychologie üblich ist, soll hier dafür plädiert werden, Aufmerksamkeit gerade in ihrem, Zwischenstatus' zu thematisieren. Hierbei ist sie als Phänomen zu charakterisieren, das sich nicht auf die eine oder andere Form reduzieren lässt, sondern sich zwischen personalen und subpersonalen Ebenen, innerer Kontrolle und äußerer Beeinflussung, passiven und aktiven Faktoren sowie, niederen' und , höheren' Stufen der Kognition befindet und zwischen diesen vermittelt. Die Kontinuität des Erlebens, die die Aufmerksamkeit dabei stiftet, lässt sich aus einer dynamischen bzw. genetisch-phänomenologischen Perspektive sichtbar machen: Hier wird Aufmerksamkeit als subjektives Phänomen bestimmt, das sich durch seine passiven, zeitlichen und situativen Dimensionen sowie insbesondere durch seine leiblichen Aspekte auszeichnet.

Im Folgenden werden die Ausganspunkte, Fragen und Thesen eines solchen dynamischen Ansatzes skizziert (Punkt 1). Hierbei wird zwischen verschiedenen Aufmerksamkeitsstufen und -strategien unterschieden, die von einer operativ fungierenden Selektion bis hin zu expliziter Fokussierung reichen. In Auseinandersetzung mit und Ergänzung zu gängigen kognitionspsychologischen Annahmen wird dabei für den Einbezug einer spezifisch leiblichen Form der Aufmerksamkeit

1 Die explizite, sekundäre Aufmerksamkeit kann dabei jederzeit durch die primäre, automatische Aufmerksamkeit unwillkürlich unterbrochen werden, z. B. durch einen neuen, besonders salienten Wahrnehmungsstimulus, vgl. Titchener (1911, S. 268ff.). 
plädiert. Weiterhin wird versucht, die Gegenstandsbereiche der Aufmerksamkeit graduell aufzufassen, von dem momentanen expliziten Fokus oder Thema bis hin zu räumlichen, zeitlichen und qualitativen Randbereichen des Wahrnehmung. Diese Randbereiche sowie die motivationale Dimension der verschiedenen Aufmerksamkeitsstufen werden in Punkt 2 als Horizonte der Aufmerksamkeit expliziert: noematische Horizonte (a) werden die gegenständlichen und thematischen Randbereiche der momentanen Aufmerksamkeit genannt, während noetische Horizonte (b) die subjektiven Motivationen impliziter und expliziter Art, wie Dispositionen, Habitualitäten und Interessen bezeichnen.

\section{$1 \quad$ Ein dynamischer Ansatz zur Untersuchung der Aufmerksamkeit}

Im Sinne eines dynamischen Ansatzes wird die Aufmerksamkeit nicht am Paradigma der punktuellen Wahrnehmung allein untersucht. Vielmehr werden zeitlich ausgedehnte Aufmerksamkeitsverläufe in ihrem Bezug zur körperlichen Beschaffenheit und zu den kinästhetischen Möglichkeiten und Fähigkeiten des Wahrnehmungssubjekts betrachtet. ${ }^{2}$ Aufmerksamkeit wird dabei weder als Ausschlussmechanismus konzipiert, der das Wahrnehmungssystem vor Überlastung schützt, noch als begrenzte Ressource, die auf bestimmte sensorische Bereiche verteilt wird. Die strukturelle Begrenztheit des Wahrnehmungsfeldes stellt jedoch keinen Nachteil dar, sondern richtet die Wahrnehmung auf handlungsrelevante Elemente aus. Eine physiologische Begrenzung der Informationsverarbeitung ${ }^{3}$ muss zudem nicht der einzige Grund dafür sein, dass eine differentielle Wahrnehmung im Sinne der Aufmerksamkeit erforderlich wird. Selektion würde auch dann eine wichtige Funktion erfüllen, wenn das perzeptive und kognitive System in der Lage wäre, alle Daten parallel und vollständig zu verarbeiten. ${ }^{4}$ Selbst ein Organismus, der über eine unbegrenzte Verarbeitungskapazität verfügte, müsste zwischen alternativen Reizen auswählen, um seine Handlungen zu steuern. ${ }^{5}$ Phänomenologisch ist daher

2 Dies betonen phänomenologische Theorien zu Bewegung, Leib und leiblichem Weltbezug sowie kognitionswissenschaftliche Ansätze, die den Zusammenhang von Kognition, Bewegung und Körperlichkeit in Form einer, embodied cognition' oder eines, enactive approach ' hervorheben, vgl. Merleau-Ponty (1945); Husserl (1952); Varela et al. (1991); O’Reagan und Noë (2001); Noë (2004); Gallagher (2005); Thompson (2007).

3 Vgl. Broadbent (1971, S. 147).

4 Vgl. Pashler (1998, S. 227, 218, 403).

5 Vgl. Neumann (1996, S. 589; 1987); Allport (1987; 1989); van der Heijden (1992). 
die Aufmerksamkeit im Hinblick auf die unterschiedlichen Erscheinungsweisen der Wahrnehmungsgegenstände in ihrer Relevanzstruktur zu betrachten, wodurch eine graduelle Auffassung entsteht, die von fokussierten bis zu hintergründigen perzeptiven Gehalten reicht. ${ }^{6}$

Im Unterschied zu der methodisch auf einfache Wahrnehmungs- und Entscheidungssituationen eingegrenzten psychologischen Experimentalsituation zeigt sich hierbei, dass sich die Aufmerksamkeit im , lebensweltlichen Handlungsalltag' nicht in erster Linie auf leere Räume, distinkte Objekte oder gar einzelne features richtet, sondern meist situativ und leiblich fungiert. Diese Komponente lässt sich anhand komplexer kinästhetischer Vollzüge, wie etwa beim Fußballspiel, illustrieren. ${ }^{7}$ Wie der französische Phänomenologe Maurice Merleau-Ponty beschreibt, ist für den ,engagierten 'Fußballspieler das Fußballfeld nicht in erster Linie ein statischer Gegenstand seiner Wahrnehmung, der unabhängig von den verschiedenen Perspektiven und perspektivischen Transformationen konstant bliebe. Das Spielfeld stellt für ihn vielmehr Aufforderungen und Möglichkeiten für Wahrnehmungen, Bewegungen und Handlungen bereit - es lädt den Spieler förmlich zum Spielen ein. Es ist für diesen von ,Kraftlinien' durchzogen und in Zonen eingeteilt, die auf mögliche Spielzüge verweisen und zu spezifischen Bewegungen Anlass geben. Das Feld kann nicht isoliert von den praktischen Intentionen des erfahrenden Subjekts wahrgenommen werden, es ist kein statischer Gegenstand, sondern konstituiert eine holistische Wahrnehmungssituation. Auf dieser Dialektik von Milieu und Handlung, wie Merleau-Ponty es nennt, beruht die spezielle Aufmerksamkeit und operativ-intentionale Bewusstseinskontinuität des Spielers. Jede seiner Bewegungen und jedes Manöver der Mannschaft modifizieren den Charakter dieses Erfahrungsfeldes und restrukturieren es. Dabei heben sich stetig neue Affordan$z e n^{8}$ vor dem räumlichen und sensomotorischen Hintergrund ab. Die Einheit der Erfahrungssituation bleibt jedoch durch die gemeinsamen Aktionen der Spieler

6 Distraktoren oder Ablenkungen der Aufmerksamkeit weisen in dieser Hinsicht nicht auf eine fehlende Verarbeitung, sondern auf eine andersartige Verarbeitung hin, die weniger expliziten Formen des Bewusstseins entspricht: „It is because we can process these stimuli that we can be distracted by them, and it is because we can be distracted by them, not because we can't process them, that we need mechanisms of attention to provide selectivity and focus" (Mole 2009, S. 503).

7 Vgl. Merleau-Ponty (1976, S. 193f.).

8 Der Begriff der Affordanzen wurde von Gibson (1979) geprägt und bezeichnet den Charakter des Angebots oder der Aufforderung, die von Gegenständen ausgehen und auf die das Subjekt bzw. der Akteur entsprechend eingehen kann. Ein Stuhl besitzt für den Menschen beispielsweise den Aufforderungscharakter einer, Sitzgelegenheit ${ }^{\star}$ - für einen anders gearteten Organismus, wie etwa eine Ameise oder auch einen Elefanten, hätte der Stuhl nicht dieselbe Signifikanz. Affordanzen sind also nicht als unabhängige 
und die kontinuierliche Wahrnehmung des Feldes erhalten bzw. wird gerade in dem temporalen Abfluss der kinästhetischen Erfahrung stets aufs Neue hergestellt.

Für einen vorbeigehenden Spaziergänger, der nicht Teil dieser Spielsituation ist, würde sich das Fußballfeld in ganz anderer Weise darstellen: Was ihm als zufälliger Beobachter in und an diesem Spielfeld auffällt, was er explizit bemerkt und was seine weitere Wahrnehmung implizit beeinflusst, wird sich erheblich von der Aufmerksamkeit des Fußballspielers unterscheiden, der auf dem Feld leiblich engagiert ist. Diese verschiedenen Aufmerksamkeitsverläufe lassen sich in Form von messbaren Blickbewegungen (Fixationen) auch experimentell erfassen. Die Art und Weise der Interaktion zwischen Subjekt und Umwelt und die damit verbundene Sicht auf die Welt lässt sich nicht nur phänomenologisch beschreiben, sondern schlägt sich auch physiologisch nieder: Sich wiederholende Wahrnehmungseindrücke, ausgeführte Tätigkeiten und erworbene Fähigkeiten hinterlassen ontogenetisch ihre Spuren, als personale und leibliche Habitualitäten sowie als plastische Modulationen im Gehirn. ${ }^{9}$

Im Rahmen einer dynamischen Sicht können nun verschiedene Stufen der Aufmerksamkeit unterschieden und in Anlehnung an den Begriff der Intentionalität als Ausprägungen einer Attentionalität ${ }^{10}$ verstanden werden, die einmal passiv-fungierend und einmal aktiv-orientierend bestimmt werden kann. Der Aufmerksamkeit wird die Funktion zugesprochen, auf beide Weisen eine Integration der zeitlichen und inhaltlichen Dimensionen der Erfahrung sowie zugleich eine selektive Strukturierung derselben zu leisten. Weiterhin ermöglicht sie dem Subjekt, sein Umfeld interessegeleitet zu erkunden, indem Affordanzen entgegengekommen wird und spezifische Informationen gesucht, gesammelt und miteinander kombiniert werden, um Wissen für den weiteren sensomotorischen Vollzug nutzen zu können.

Die basale Dimension der Attentionalität bilden Empfindungen und passivsituative Gestaltverläufe, also integrativ-synthetische Leistungen, die eine zeitlich und inhaltlich kohärente Wahrnehmung ermöglichen. Auf den weiteren Stufen kommt es dann zu einem Anstieg an Differenziertheit. Die Selektion bezieht sich zunehmend auf gegenständliche Zusammenhänge und bildet einen diskreten Fokus aus. Im Rahmen dieser expliziten Aufmerksamkeit treten die integrativen Funktionen bewusstseinsmäßig in den Hintergrund, werden unthematisch, spielen aber weiterhin für den Wahrnehmungsvorgang eine zentrale Rolle. Sie erlauben nämlich das gemeinsame (kolligierende) Auffassen von mehreren räumlich getrennten oder

objektive Gegebenheiten zu verstehen, sondern in Relation zur physischen, behavioralen und kognitiven Struktur des jeweiligen auf sie reagierenden Organismus.

9 Vgl. zum Paradigma der neuronalen Plastizität z. B.: Buonomano und Merzenich (1998); Draganski et al. (2006); Davidson und Lutz (2007).

10 Vgl. Breyer (2011). 
inhaltlich verschiedenen Objekten, indem sie diese durch ein aufrechterhaltenes Wahrnehmungsinteresse zu einer thematischen Ausrichtung verbinden.

Eine elementare Funktion der Aufmerksamkeit besteht demnach in einer Differenzierung des Sinnesfeldes. ${ }^{11}$ Durch diesen sensorischen Bezug zur Welt entstehen erste Formen von, Abgehobenheiten ' im Erfahrungsfeld, die das Subjekt affizieren. ${ }^{12}$ Die Rezeptivität des Subjekts besagt hier kein passives Ausgeliefertsein an Eindrücke, sondern impliziert bereits einen selektiven Bezug zur Welt, der durch passive Faktoren der individuellen Erfahrungsgeschichte oder durch die aktuellen leiblichen Tätigkeiten motiviert ist. Diese Selektivität hat integrative Bedeutung, da neue Erscheinungen in den Erfahrungshaushalt integriert werden und so einerseits zu einem zeitlich und thematisch einheitlichen Wahrnehmungsverlauf führen und andererseits eine bekannte Lebenswelt für das Subjekt entstehen lassen, die fortwährend angereichert wird. Sinkt hingegen das Interesse an einem Objektbereich wieder ab, so wird auch prospektiv die Aufmerksamkeit weniger von derartigen Elementen affiziert. Insofern erfüllt die Aufmerksamkeit für das Bewusstsein und die Erfahrung schon auf der Wahrnehmungsebene eine ,ökonomische' Funktion. ${ }^{13}$

Gewiss ist der Leib als Sensorium und grundlegendes Medium des subjektiven Weltbezugs schon auf der genannten Ebene operativ. Von einer leiblichen Aufmerksamkeit im stärkeren Sinne können wir aber insbesondere dort sprechen, wo körperlich orientierte Handlungen sichtbar werden, wie etwa dem Gehen, Fußballspielen, Tanzen etc. Hier lässt sich zwischen angeborenen und erlernten leiblichen Fähigkeiten unterscheiden sowie zwischen aktuellen, reizbasiert parametrisierten Bewegungsverläufen und typischen habituellen Reaktionsformen. Die Aufmerksamkeit, wie sie sich in der leiblichen Reaktivität und Spontaneität zeigt, ist dem ausführenden Subjekt häufig nicht in derselben Form thematisch wie höhere kognitive Prozesse. Dennoch lässt sie sich etwa in der körperlichen Spannung, der spezifischen Ausrichtung auf Handlungsrelevantes und der Wachsamkeit in Bezug auf die umgebenden Ereignisse feststellen.

Von diesen eher passiv-dynamischen Aspekten der Aufmerksamkeit können explizite Aufmerksamkeitsstrategien unterschieden werden, die sich auf ein spezielles Thema oder eine abgegrenzte Handlung beziehen - etwa die Fokussierung auf einen Gegenstand oder Ort zum Zweck der näheren Betrachtung, die Suche von

11 In ähnlicher Weise wie in der genetischen Phänomenologie nimmt man auf neurobiologischer Ebene ebenfalls zeitliche, Synthesen“ als Grundlage von Wahrnehmung an: Hier findet eine Phasensynchronisation und eine „large-scale integration“ statt, vgl. Varela et al. (2001); Singer (2007).

12 Husserl (1966b, S. 168) spricht von einem „affektiven Relief“.

13 Vgl. zum Begriff einer „Ökonomie der Aufmerksamkeit“ im Bereich der Intersubjektivität und Medialität, der über die hier gemeinte Perzeptualität hinausreicht Franck (1998). 
etwas Bestimmtem oder die Ausführung geplanter Handlungen, die Konzentration erfordern, ebenso die kognitive Konzentration auf einen Text, ein Gespräch oder einen Gedanken. Diese thematische Aufmerksamkeit zeichnet sich meist dadurch aus, dass eine einzelne Sinnesmodalität überwiegt. Während die passiv-synthetischen Prozesse eine globale und kontextuelle Wahrnehmung erzeugen und somit die integrative Funktion im Vordergrund steht, tritt hier die selektive, gleichsam analytische $e^{14}$ Funktion der Aufmerksamkeit zutage.

Ergänzend zu den Unterscheidungen in passiv-latente und aktiv-patente, fungierende und thematische sowie synthetische und analytische Aufmerksamkeit geht es im Folgenden um die Kontextualität bzw. Horizontalität der Aufmerksamkeit. Der Kerngedanke hierbei ist, dass sich kein attentionaler Akt hinreichend beschreiben lässt, wenn man ihn isoliert betrachtet, sondern dass seine Eingebettetheit in subjektive, zeitliche und gegenständliche Zusammenhänge in die phänomenologische Analyse einbezogen werden muss.

\section{Horizonte der Aufmerksamkeit}

\subsection{Noematische Horizonte}

In der empirischen Aufmerksamkeitsforschung finden die Aspekte der Peripherie, des Hintergrundbewusstseins und der Horizonte des aktuellen Aufmerksamkeitsfokus häufig nicht genügend Beachtung. ${ }^{15}$ Diese Faktoren bezeichnen zunächst den Sachverhalt, dass es zwischen Bewusstsein und Nicht-Bewusstsein eines visuellen Umfeldes Klarheits- und Intensitätsgradienten gibt. Das jeweilige Thema der Aufmerksamkeit lässt sich dabei nur in Relation dazu erkennen, was sich gerade nicht im Fokus befindet, sondern im Hintergrund, als nebenbei oder gerade eben Wahrgenommenes erfahren wird.

Das von Aron Gurwitsch beschriebene Thema der Aufmerksamkeit ist auf ein thematisches Feld verwiesen, das sich von einem bloßen Hintergrund oder Rand des Bewusstseins dadurch unterscheidet, dass es mit dem Thema strukturell und

14 Vgl. Merleau-Ponty (1966, S. 30).

15 Arvidson kritisiert die mangelnde Berücksichtigung von Kontexten in der empirischen Aufmerksamkeitsforschung. Unter kontextuellen Faktoren versteht er bottom-up Kriterien, die das gegenständliche Umfeld prägen, vgl. Arvidson (2008, S. 540f.; 2010). Vgl. für eine ausführliche phänomenologische Darstellung der attentionalen Horizonte Wehrle (2013). 
inhaltlich verbunden ist. ${ }^{16}$ Gurwitsch stellt mit Hilfe der Gestalttheorie dar, dass Thema und thematisches Feld nicht unabhängig voneinander existieren, sondern einen Strukturzusammenhang bilden, aus dem sie sich erst retrospektiv abstrahieren und einzeln betrachten lassen können. Objekte und ihre noematischen (d.h. gegenständlichen) Kontexte beeinflussen sich insofern wechselseitig in Form einer gestalthaften Strukturierung. Darüber hinaus stellt das thematische Feld einen inhaltlichen Horizont dar, der den Bereich des Visuellen überschreiten kann, indem er beispielsweise assoziativ Erinnerungen weckt. Der objektiv messbare visuelle Fokus und dessen Peripherie stimmen insofern nie ganz mit dem subjektiven Thema der Aufmerksamkeit und seinem Umfeld überein.

Wie wichtig es ist, gegenständliche und inhaltliche Horizonte in die Untersuchung der Aufmerksamkeit mit einzubeziehen, lässt sich empirisch deutlich machen, sobald man die Untersuchung nicht mehr auf die Suche nach einfachen Stimuli wie geometrischen Objekten beschränkt. Konfrontiert man Probanden mit komplexeren Szenen, etwa einer belebten Straße, lässt sich die beteiligte Aufmerksamkeit nicht nur von einzelnen Stimuli lenken. Eine solche Szene bietet eine Vielzahl von Details, die ein Betrachter nicht alle im Einzelnen und in gleicher Klarheit oder Intensität auffassen kann. Stattdessen wird ein grober Gesamteindruck gebildet, der es erlaubt, die Bedeutung der Szene auf den ersten Blick intuitiv zu erkennen. ${ }^{17}$ Anstatt einer fokal bzw. lokal ausgerichteten Aufmerksamkeit, wie sie etwa bei der Suche nach bestimmten Objekten vorkommt, lässt sich hier vielmehr eine globale Aufmerksamkeitsstrategie erkennen. Diese ist besonders relevant für eine dynamische Sicht der Aufmerksamkeit, weil in der alltäglichen Wahrnehmung einzelne Objekte nicht isoliert vorkommen, sondern immer schon Teil eines Verweisungszusammenhanges sind. Wie dynamisch die horizontale Wahrnehmungsstruktur ist, zeigt sich etwa beim Autofahren. Hier ist nicht nur eine Selektion im Hinblick auf den unmittelbaren Straßenverlauf notwendig, sondern in gleichem Maße eine periphere Aufmerksamkeit auf andere Fahrzeuge und vorausliegende Straßenschilder.

Für das Verständnis einer Situation oder Szene muss dabei über den gegenständlichen Kontext hinaus auch die inhaltliche Verbindung zum Wahrnehmungs- oder Handlungsakt und dem wahrnehmenden Subjekt sowie seinen Interessen berücksichtigt werden. Das thematische Feld kann in diesem Sinne von dem rein visuellen Mittelpunkt einer Szene abweichen. Betrachtet man eine Szene oder Situation, bemessen sich die visuellen Fixationen häufig nicht nach dem räumlichen, sondern dem inhaltlichen Mittelpunkt, d.h. den bedeutungsrelevanten Aspekten wie etwa Gesichter oder Objekte und Regionen, die vom Subjekt als informativ eingestuft

16 Vgl. Gurwitsch (1929).

17 Vgl. Greene und Oliva (2009); Biederman et al. (1974). 
werden..$^{18}$ Ein passender semantischer Kontext einer Szene kann denn auch die Suche oder Identifikation eines Zielobjekts beschleunigen. Ein Feuerhydrant, der auf einem Briefkasten steht, oder ein Sofa, das in der Luft schwebt, wird innerhalb einer Szene nicht so schnell entdeckt. ${ }^{19}$ Im semantischen Kontext einer Küche lässt sich dagegen eine Brotbüchse leichter identifizieren als etwa eine Trommel. ${ }^{20}$ Hieran zeigt sich, dass die Aufmerksamkeit responsiv ist gegenüber der sinnhaften Strukturierung der Wahrnehmungswelt.

Die Bedeutung von Kontexten für die Erfahrung erweist sich darin, dass wiederholt auftretende Objektkonfigurationen und Umweltsituationen direkt perzipiert werden und so eine gewisse Invarianz und Stabilität gewährleisten. Die Information über typisch gegenständlich-räumliche Kontexte erleichtert die Orientierung und sorgt dafür, dass nicht jedes Objekt einzeln fokussiert werden muss. Informationen über invariante räumliche und gegenständliche Strukturen werden implizit in der Erfahrung erlernt. ${ }^{21}$ Zielobjekte, die dann in einem solchen invarianten Kontext auftreten, können so schneller lokalisiert werden. Auch dynamische Kontexte, die auf der Veränderung von Gegenständen basieren, werden habitualisiert und für die Orientierung des weiteren Verhaltens genutzt: Profis in Teamsportarten erwerben beispielsweise ein intuitives Gespür dafür, wie sich das gesamte Feld der Mitspieler bewegt und welche typischen Relationen von Umfeld und Zielobjekt (Ball) sich für die bestimmte Spielsituationen ergeben. ${ }^{22}$

\subsection{Noetische Horizonte}

Ein Phänomen, das innerhalb der psychologischen und phänomenologischen Aufmerksamkeitsforschung immer wieder auftaucht, ist, dass (für das jeweilige Subjekt) besonders relevante Stimuli Aufmerksamkeit erregen bzw. einen Wechsel der vorangegangenen Aufmerksamkeit motivieren können. Ein bekanntes Beispiel hierfür ist der Cocktail-Party-Effekt ${ }^{23}$, also das Hören des eigenen Namens

18 Vgl. Loftus und Mackworth (1978); Mackworth und Morandi (1967); Henderson (2003).

19 Vgl. Palmer (1975).

20 Vgl. Biedermann et al. (1982).

21 Dies zeigen u. a. Versuche mit dem contextal cueing Paradigma, vgl. Chun (2000).

22 Für das implizite Lernen von kontextueller Information und die entsprechenden habituellen Erinnerungsleistungen scheint der Hippocampus besonders relevant zu sein. Im Gegensatz zum deklarativen Gedächtnis geht man bei kontextuellen Lernprozessen davon aus, dass dabei sogenannte implizite Repräsentationen gebildet werden. Vgl. Chun (2003).

23 Vgl. Cherry (1953). 
in geselliger Atmosphäre mit hohem Geräuschpegel, das einen plötzlich aus dem Gespräch herausreißen kann, in das man vertieft ist und bei dem man ansonsten erfolgreich das Hintergrundrauschen ausblendet. Gleiches gilt für Reize, die in einem inhaltlichen oder assoziativen Zusammenhang mit unseren Handlungen oder soeben Wahrgenommenem stehen. Semantik und subjektive Relevanz sprechen als top-down Faktoren gegen die Interpretation, dass Aufmerksamkeit allein extrinsisch, d.h. durch objektive Reize angezogen und gelenkt wird.

Welche Umstände führen nun aber zu einem expliziten Aufmerksamkeitswechsel und welche Rolle spielen hierbei die jeweiligen subjektiven Relevanzkriterien und das aktuelle Engagement des attentionalen Subjekts? Solche Fragen sind im Hinblick auf alltägliche Wahrnehmung, wie etwa im Straßenverkehr, äußerst wichtig, vor allem dann, wenn es zu Gefahrensituationen kommt. ${ }^{24}$ Würden neue und plötzlich auftretende Stimuli automatisch unsere Aufmerksamkeit auf sich ziehen, dann würden wir ein auf die Straße rennendes Kind unmittelbar bemerken und könnten besser ausweichen. Auch wenn solche aufgrund von Bewegung oder Kontrast salienten Merkmale das Bemerken generell erleichtern, hängt viel auch von den selektiven Relevanzkriterien bzw. dem sogenannten attentional set ab: „[T]he unexpected objects that people consciously see depend on the ways in which they ,tune their attention for processing of specific types of stimuli-that is, on the attentional set. ${ }^{\text {" } 25}$

Neben einem aufgabenspezifischen attentional set, das die Erwartungen und Antizipationen in der jeweiligen Wahrnehmungssituation bestimmt, müssen noch andere noetische Komponenten wie emotionale und normative Einstellungen, Interessen sowie habituelle und leibliche Vermögen einbezogen werden. Sie fungieren in gleichem Maße als Motivationen für Blickbewegungen und Fixationshäufigkeiten innerhalb einer beobachteten Szene. Fixationsreihenfolgen der Probanden auf entsprechende Objekte innerhalb einer Straßenszene unterscheiden sich etwa maßgeblich, je nachdem ob sie Automechaniker, Architekten oder Straßenbauer von Beruf sind. Solche Berufsinteressen gehen mit spezifischen lebensweltlichen Erfahrungen und erlerntem Wissen im jeweiligen Bereich einher. All dies kann

24 Vgl. Most et al. (2005, S. 218).

25 In einem entsprechenden Experiment, das Fahrsituationen auf einem visuellen Display simulierte, wurden über eine längere Zeit entweder gelbe oder blaue Straßenschilder eingeblendet. Assoziierten die Probanden die Farbe,gelb ' mit relevanten Informationen (Schilder), so wurde auch ein plötzlich von der Seitenstraße kommendes gelbes Motorrad im Vergleich zu einem blauen schneller erkannt, vgl. Most und Astur (2007). 
nachweislich zu spezialisierten impliziten und expliziten Antizipationen in Bezug auf eine Situation führen, die den Wahrnehmungsverlauf entsprechend lenken. ${ }^{26}$

Dies wird besonders auf der Ebene der leiblichen Aufmerksamkeit deutlich: Das antizipative Wahrnehmungsverhalten von Profisportlern unterscheidet sich auf diese Weise von Nicht-Sportlern oder Anfängern. Im Gegensatz zu normalen Personen bemerken Sie etwa semantische Veränderungen in einem change blindness Experiment viel öfter, wenn entsprechende Bilder aus ihrer Sportart verwendet werden. ${ }^{27}$ Die Wahrnehmung von Experten geht über das aktuell Präsentierte hinaus, indem es automatisch bekannte Situationen nach bisherigen Erfahrungswerten vervollständigt. Die habituell erworbene passive Antizipation weiterer Bewegungsabläufe beeinflusst die Beurteilung der aktuellen Wahrnehmung. Hier kann es bei Profis dann auch zu Fehlbeurteilungen kommen, da sie im Einzelfall nicht zwischen dem unterscheiden können, was gerade tatsächlich präsentiert wird, und dem, was sie virtuell ergänzen.

Die selektive Relevanzstruktur hängt weiterhin davon ab, ob man eine Szene lediglich beobachtet oder aktiv in eine Handlung involviert ist. Eine Bewegung, die man gerade ausführt, kann beeinflussen, wie man die Bewegungen anderer Personen wahrnimmt. Die erworbenen subjektiv-habituellen Bewegungsmuster und leiblichen Fertigkeiten bestimmen ebenfalls mit, was uns an den Bewegungen anderer jeweils auffällt. Die Entdeckung der sogenannten „Spiegelneuronen“ legte in diesem Zusammenhang die Vermutung nahe, dass dieselben Muster bei der Beobachtung und bei der eigenen Ausübung bestimmter Bewegungen am Werk seien. ${ }^{28}$ Neben einer vermeintlich sozialen Bedeutung für die Empathie des Menschen scheinen die Spiegelneuronen eine Art „motorische Resonanz“ zwischen dem engagierten Beobachter und demjenigen zu erzeugen, der ihm bekannte Bewegungen ausübt. Der Beobachtende weist etwa eine höhere Sensitivität auf für Situationen, die mit Bewegung und Aktivitäten verbunden sind, die ihm habituell geläufig sind oder aber Ähnlichkeiten zu den eigenen aktuellen Bewegungsabläufen aufweisen. ${ }^{29}$

Ein besonders prägnantes Beispiel für den Einfluss solcher leiblich-habitueller Horizonte auf die Wahrnehmung stellen Experimente dar, die die Aufmerksamkeit

26 Vgl. Enns und Lleras (2008); Rock (1977); Iordanescu et al. (2008); Balas und Sinha (2007).

27 Vgl. Werner und Thies (2000).

28 Darin sehen Kognitionswissenschaftler und einige Phänomenologen ein physiologisches Indiz für die neuronale Verankerung sozial-kognitiver Leistungen, vgl. Rizzolatti et al. (2001); Schütz-Bosbach und Prinz (2007); Gallagher und Zahavi (2008, S. 181f.). Mittlerweile scheint das Interesse an den Spiegelneuronen jedoch aus vorwiegend methodologischen Gründen beträchtlich geschwunden zu sein.

29 Schütz-Bosbach und Prinz (2007, S. 349). 
von Personen mit spezialisierten motorischen Fähigkeiten untersuchen. Es zeigt sich hierbei eine ausgeprägte Resonanz in Bezug auf Situationen oder Personen, die dasselbe Expertengebiet teilen. Bei Personen, die beruflich viele Texte tippen müssen, wurde etwa eine entsprechende motorische Aktivierung festgestellt, sobald man ihnen einen Buchstaben nur zeigte. ${ }^{30}$ Bei Ballett- und Capoeiratänzern lassen sich ähnliche physiologische Effekte nachweisen, wenn sie Tänzer ihrer eigenen Disziplin im Vergleich zu Tänzern anderer Tanzstile beobachten. Auf der Verhaltensebene korrespondiert dies mit besseren Diskriminierungsleistungen, die bei gewohnten und bekannten Bewegungen erbracht werden. ${ }^{31}$

All diese Beispiele deuten auf die Wichtigkeit noetischer (subjektiver) Horizonte für die Aufmerksamkeitslenkung hin. Diese Horizonte ermöglichen nicht nur prinzipiell eine kohärente Erfahrung der Welt über die Zeit hinweg, sondern tun dies auf je individuelle Weise. Zugleich ist das selektive Wesen der menschlichen Wahrnehmung bis in seine tiefsten habituellen Erwerbe und Motivationen hinein von seinem intersubjektiven und kulturellen Umfeld durchdrungen. Der selektive Aufmerksamkeitsstil bildet sich von Beginn an in und durch die Interkationen mit den Mitmenschen und seiner Umwelt. Zwischen inneren und äußeren Einflüssen kann insofern nur schematisch oder im experimentellen Einzelfall unterschieden werden. Als dynamische Motivationsgrundlagen der Aufmerksamkeit kann der Horizontbegriff und mehrere Dimensionen aufgefaltet werden:

Der passiv-zeitliche Horizont beinhaltet die mit dem gegenwärtig Präsenten unmittelbar oder mittelbar verknüpften zeitlichen Erfahrungselemente. Hierzu gehören zum einen die retentionalen und protentionalen Inhalte ${ }^{32}$, die die erlebte

30 Vgl. Rieger (2004).

31 Vgl. Calvo-Merino et al. (2006; 2010).

32 Retention und Protention bilden nach Husserl zusammen mit dem erlebten Jetzt (Impression) das Zeitfeld eines jeden gegenwärtigen Erlebnisses. Husserl erläutert dies am Beispiel des Hörens einer Melodie: Der jetzt gehörte Ton wandelt sich im Bewusstsein unmittelbar in einen soeben-gewesenen Ton, die Retention des vormaligen Jetzt-Tones. Mit Retention ist dabei nicht der reale Nachhall eines Tones gemeint, sondern vielmehr der Umstand, dass der zuvor empfundene Jetzt-Ton noch immer im Bewusstsein präsent ist, aber nun in modifizierter Weise. Das aktuelle impressionale Bewusstsein geht so „ständig fließend über in immer neues retentionales Bewusstsein“, wie Husserl sagt. Nur weil der vorangegangene Ton noch retentional im Bewusstsein ist, erscheint der folgende Ton als Teil einer kontinuierlichen Melodie und nicht als isoliertes Geräusch. Auf der Basis aller Retentionen kann sich in die andere ,Zeitrichtung eine Protention aufbauen, die beim Hören einer Melodie den nächsten Ton antizipiert - nicht in der Weise einer Vergegenwärtigung, sondern als implizite Tendenz. Solange ein Ton oder eine Melodie erklingt, gehört zum zeitkonstituierenden Prozess also nicht nur das Festhalten des Erlebten im Bewusstsein, sondern auch eine Gerichtetheit auf das Noch-nicht-Erlebte. Im Ablauf einer Zeitwahrnehmung stellt sich damit „immerfort ,Erwartung“ ein, wie 
Gegenwart mitkonstituieren, und zum anderen sedimentierte und antizipierte Inhalte, die qua Repräsentation und Antizipation zugänglich sind.

Der leiblich-habituelle Horizont wird durch motorische Erwerbe, Fertigkeiten und habituelle Verhaltensmuster gebildet, die die Wahrnehmung von Situationen und Bewegungsverläufen beeinflussen. Er entspricht dem nicht-thematischen Wahrnehmungshabitus, der automatische Orientierung ermöglicht, indem er die Umgebung in gewohnte und bekannte Wege strukturiert.

Der personale Interessenshorizont umfasst handlungsleitende Interessen, Lebensentwürfe, Expertisen, Vorlieben und Bedürfnisse. Diese sind meist nicht vollständig in ihrer Wirkung auf die alltägliche Wahrnehmung thematisch, aber einer nachträglichen Reflexion prinzipiell zugänglich. Der personale Interessenhorizont prägt von daher Identität des Subjekts wesentlich mit und kann zum Gegenstand von Selbstvergegenwärtigungen werden.

Der lebensweltliche Horizont, oder schlicht die Lebenswelt, stellt den intersubjektiven und soziokulturellen Rahmen des personalen Interessenshorizontes dar. Normen und Traditionen gestalten hier von Beginn an die subjektive Rezeptivität und Responsivität gegenüber Affizierendem mit und bestimmen weitere Aufmerksamkeitsausrichtungen.

Zusammengefasst lässt sich sagen: In der Frage nach den noematischen Horizonten geht es darum aufzuklären, wie die Dinge, die man passiv bemerkt oder auf die man aktiv aufmerkt, mit anderen Dingen in gegenständlicher, gestalthafter und bedeutungsmäßiger Beziehung stehen. Die Frage nach den noetischen Horizonten befasst sich demgegenüber einerseits damit, warum ein Subjekt in einem speziellen Fall gerade dies und nicht jenes bemerkt, und andererseits, ob sich typische Aufmerksamkeitsstile bestimmen lassen, mit deren Hilfe sich voraussagen lässt, worauf bestimmte Personen eher aufmerksam werden als andere.

Husserl betont. Weder das Festhalten im Bewusstsein und dessen modifizierter Inhalt, noch die Antizipation und dessen protentionaler Inhalt (der antizipierte Ton) dürfen dabei als explizite kognitive Vorgänge gedeutet werden. Das Zeitbewusstsein gilt bei Husserl als Grundform der passiven Synthesis, die die formale Organisation des Bewusstseins darstellt. Eine Retention ist dabei keine Erinnerung und eine Protention keine (explizite) Erwartung, beide sind notwendiger Bestandteil der aktuellen Wahrnehmung. Sie garantieren deren Kontinuität, d.h. die Integration aller Sinnesinhalte in einen einheitlichen Zeit- und Erlebnisstrom. Vgl. Husserl (1966a, S. 29f.; 2001, S. 7). 


\section{Literatur}

Allport, D. A. (1987). Selection for action: some behavioral and neurophysiological considerations of attention. In H. Heuer, \& A. F. Sanders (Hrsg.), Perspectives on perception and action (S. 395-419). Hillsdale, NJ: Erlbaum.

Allport, D. A. (1989). Visual attention. In M. I. Posner (Hrsg.), Foundations of cognitive science (S. 631-682). Cambridge, MA: MIT Press.

Arvidson, P. S. (2008). Attentional capture and attentional character. Phenomenology and the Cognitive Sciences 7, 539-562.

Arvidson, P. S. (2010). Attention in context. In: S. Gallagher, \& D. Schmicking (Hrsg.), Handbook of phenomenology and cognitive science (S. 99-123). Dordrecht: Springer.

Balas, B., \& Sinha, P. (2007). Filling in colour in natural scenes. Visual Cognition 15, 765-778.

Biederman, I., Rabinowitz, J. C., Glass, A. L., \& Stacy, E. W. (1974). On the information extracted from a glance at a scene. Journal of Experimental Psychology 103, 597-600.

Biederman, I., Mezzanotte, R. J., \& Rabinowitz, J. C. (1982). Scene perception: detecting and judging objects undergoing relational violations. Cognitive Psychology 14, 143-177.

Broadbent, D. E. (1971). Decision and stress. New York: Academic.

Breyer, T. (2011). Attentionalität und Intentionalität. Grundzüge einer phänomenologischkognitionswissenschaftlichen Theorie der Aufmerksamkeit. Paderborn: Wilhelm Fink.

Buonomano, D. V., \& Merzenich, M. M. (1998). Cortical plasticity: from synapses to maps. Annual Review of Neuroscience 21, 149-186.

Calvo-Merino, B., Grézes, J., Glaser, D. E., Passingham, R. E., \& Haggard, P. (2006). Seeing or doing? Influence of visual and motor familiarity in action observation. Current Biology 16, 1905-1910.

Calvo-Merino, B., Ehrenberg, S., Leung, D., \& Haggard, P. (2010). Experts see it all: configural effects in action observation. Psychological Research 74, 400-406.

Cherry, E. C. (1953). Some experiments on the recognition of speech, with one and with two ears. Journal of the Acoustical Society of America 25, 975-979.

Chun, M. M. (2000). Contextual cueing of visual attention. Trends in Cognitive Sciences 4, $170-177$.

Chun, M. M. (2003). Scene perception and memory. In D. Irwin \& B. Ross (Hrsg.), Psychology of Learning and Motivation: Advances in Research and Theory (S. 79-108). San Diego, CA: Academic Press.

Davidson, R. J., \& Lutz, A. (2007). Buddha's brain: neuroplasticity and meditation. IEEE Signal Processing 176, 172-174.

Draganski, B., Gaser, C., Kempermann, G., Kuhn, H. G., Winkler, J., Büchel, C., \& May, A. (2006). Temporal and spatial dynamics of brain structure changes during extensive learning. Journal of Neuroscience 27, 6314-6317.

Ennen, E. (2003). Phenomenological coping skills and the striatal memory system. Phenomenology and the Cognitive Sciences 2, 299-325.

Enns, J. T., \& Lleras, A. (2008). What's next? New evidence for prediction in human vision. Trends in Cognitive Science 12, 327-333.

Franck, G. (1998). Ökonomie der Aufmerksamkeit. München, Wien: Hanser.

Gallagher, S. (2005). How the body shapes the mind. Oxford: Oxford University Press.

Gallagher, S., \& Zahavi, D. (2008). The phenomenological mind. London, New York: Routledge. Gibson, J. J. (1979). The ecological approach to visual perception. Boston: Houghton Mifflin. 
Greene, M. R., \& Oliva, A. (2009). Recognition of natural scenes from global properties: seeing the forest without representing the trees. Cognitive Psychology 58/2, 137-176.

Gurwitsch, A. (1929). Phänomenologie der Thematik und des reinen Ich. Psychologische Forschungen 12, 279-381.

Heijden, A. H. C. van der (1992). Selective attention in vision. London: Routledge.

Henderson, J. M. (2003). Human gaze control during real-world scene perception. Trends in Cognitive Science 7, 498-504.

Husserl, E. (1952). Ideen zu einer reinen Phänomenologie und phänomenologischen Philosophie. Zweites Buch. Phänomenologische Untersuchungen zur Konstitution. Husserliana IV. Den Haag: Nijhoff.

Husserl, E. (1966a). Zur Phänomenologie des inneren Zeitbewusstseins. Husserliana X. Den Haag: Nijhoff.

Husserl, E. (1966b). Analysen zur passiven Synthesis. Husserliana XI. Den Haag: Nijhoff.

Husserl, E. (2001). Die Bernauer Manuskripte über das Zeitbewusstsein (1917/18). Husserliana XXXIII. Dordrecht: Kluwer.

Iordanescu, L., Guzman, E., Grabowecky, M., \& Suzuki, S. (2008). Characteristic sounds facilitate visual search. Psychonomic Bulletin Review 15, 548-554.

Loftus, G. R., \& Mackworth, N. H. (1978). Cognitive determinants of fixation location during picture viewing. Journal of Psychology: Human Perception and Performance 4, 565-572.

Mackworth, N. H., \& Morandi, A. J. (1967). The gaze selects informative details within pictures. Perception and Psychophysics 2, 547-552.

Merleau-Ponty, M. (1945). Phénoménologie de la perception. Paris: Gallimard.

Merleau-Ponty, M. (1966). Phänomenologie der Wahrnehmung. Berlin, New York: De Gruyter.

Merleau-Ponty, M. (1976). Die Struktur des Verhaltens. Berlin, New York: De Gruyter.

Mole, C. (2009). Attention. In J. Symons, \& P. Calvo (Hrsg.), The Routledge companion to philosophy of psychology (S. 495-509). London, New York: Routledge.

Most, S. B., Scholl, B. J., Clifford, E. R., \& Simons, D. J. (2005). What you see is what you set: sustained inattentional blindness and the capture of awareness. Psychological Review $112,217-242$.

Most, S. B., \& Astur, R. S. (2007). Feature-based attentional set as a cause of traffic accidents. Visual Cognition 15, 125-132.

Neumann, O. (1987). Beyond capacity: a functional view of attention. In H. Heuer, \& A. F. Sanders (Hrsg.), Perspectives on perception and action (S. 361-399). Hillsdale, NJ: Erlbaum.

Neumann, O. (1990). Visual attention and action. In O. Neumann, \& W. Prinz (Hrsg.), Relationships between perception and action (S. 227-264). Heidelberg: Springer.

Neumann, O. (1996). Theorien der Aufmerksamkeit. In O. Neumann, \& A. F. Sanders (Hrsg.), Aufmerksamkeit. Göttingen, Bern, Toronto, Seattle: Hogrefe.

Noë, A. (2004). Action in perception. Cambridge, MA: MIT Press.

O’Reagan, J. K., \& Noë, A. (2001). A sensorimotor approach to vision and visual consciousness. Behavioral and Brain Sciences 24, 939-1031.

Oliva, A., \& Torralba, A. (2006). Building the gist of a scene: the role of global image features in recognition. Progress in Brain Research: Visual Perception 155, 23-36.

Palmer, S. E. (1975). The effects of contextual scenes on the identification of objects. Memory and Cognition 3, 519-526.

Pashler, H. E. (1998). The psychology of attention. Hove: Psychology Press.

Rieger, M. (2004). Automatic keypress activation in skilled typing. Journal of Experimental Psychology: Human Perception and Performance 30, 555-565. 
Rizzolatti, G., Fogassi, L., \& Gallese, V. (2001). Neurophysiological mechanisms underlying the understanding and imitation of action. Nature Reviews Neuroscience 2, 661-670.

Rock, I. (1977). In defense of unconscious inference. In W. Epstein (Hrsg.), Stability and constancy in visual perception: mechanisms and processes (S. 321-373). New York: Wiley.

Schütz-Bosbach, S., \& Prinz, W. (2007). Perceptual resonance: action-induced modulation of perception. Trends in Cognitive Science 11, 349-355.

Singer, W. (2007). Large-scale temporal coordination of cortical activity as a prerequisite for conscious experience. In M. Velmans, \& S. Schneider (Hrsg.), The Blackwell companion to consciousness (S. 605-615). Oxford: Oxford University Press.

Titchener, E. B. (1911). A text-book of psychology. New York: Macmillan.

Thompson, E. (2007). Mind in life. Cambridge, MA: Harvard University Press.

Varela, F. J., Thompson, E., \& Rosch, E. (1991). The embodied mind. Cambridge, MA: MIT Press.

Varela, F. J., Lachaux, J. P., Rodriguez, E., \& Martinerie, J. (2001). The brainweb: phase sychronization and large-scale integration. Nature Reviews Neuroscience 2, 229-239.

Wehrle, M. (2013). Horizonte der Aufmerksamkeit. Entwurf einer dynamischen Konzeption der Aufmerksamkeit aus phänomenologischer und kognitionspsychologischer Sicht. Paderborn: Wilhelm Fink.

Werner, S., \& Thies, B. (2000). Is ,change blindness' attenuated by domain-specific expertise? An expert-novices comparison of change detection in football images. Visual Cognition 7, 163-173. 